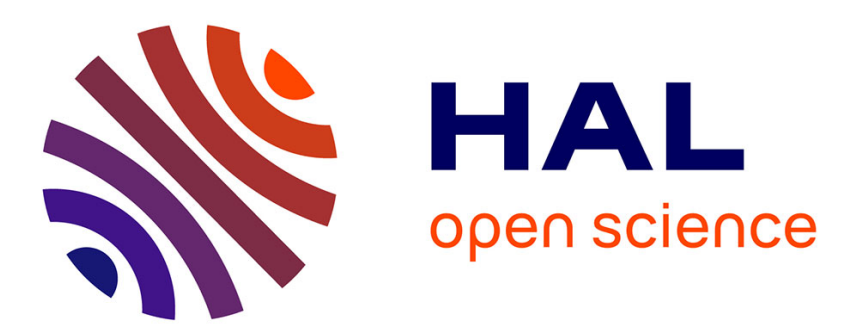

\title{
Inscription spatiale d'une activité cognitive collective de représentation de l'espace
}

\author{
Christian Brassac, Florence Le Ber
}

\section{To cite this version:}

Christian Brassac, Florence Le Ber. Inscription spatiale d'une activité cognitive collective de représentation de l'espace. Intellectica - La revue de l'Association pour la Recherche sur les sciences de la Cognition (ARCo), 2005, 41-42, pp.181-200. halshs-00140584

\section{HAL Id: halshs-00140584 https://shs.hal.science/halshs-00140584}

Submitted on 10 Apr 2007

HAL is a multi-disciplinary open access archive for the deposit and dissemination of scientific research documents, whether they are published or not. The documents may come from teaching and research institutions in France or abroad, or from public or private research centers.
L'archive ouverte pluridisciplinaire HAL, est destinée au dépôt et à la diffusion de documents scientifiques de niveau recherche, publiés ou non, émanant des établissements d'enseignement et de recherche français ou étrangers, des laboratoires publics ou privés. 
Brassac, Ch., Le Ber, F. (2005). Inscription spatiale d'une activité cognitive collective de représentation de l'espace, Intellectica 41-42, 181-200.

\title{
Inscription spatiale d'une activité cognitive collective de représentation de l'espace
}

\author{
Christian BRASSAC* - Florence LE BER**
}

\begin{abstract}
RÉSUMÉ. Rassemblés autour de la conception d'un système à bases de connaissances, des agronomes et des informaticiens travaillent collectivement à la représentation de l'organisation spatiale de territoires d'exploitation agricole. Cette représentation d'un certain type d'espace est une activité collaborative qui s'inscrit elle-même dans un espace ; cette activité collaborative est marquée par une histoire interactionnelle dont les ressources sont aussi bien des expertises, des documents, des instruments que des supports de traçage. Adoptant une approche relevant du paradigme de la cognition située et distribuée, nous considérons l'interaction comme un modelage de formes langagières, gestuelles et artefactuelles. Nous montrons comment la mobilisation de ces ressources cognitives, corporelles, documentaires et matérielles permet à l'équipe-projet de mener à bien un travail qui articule quatre espaces : l'espace agricole, l'espace de travail du groupe, l'espace d'inscriptions et l'espace numérique, qui constitue le point de mire du projet.
\end{abstract}

Mots clés : acquisition de connaissance, représentation de l'espace, interaction, artefacts, cognition située et distribuée

AbSTRACT. Spatial embedding of a collective cognitive activity for spatial representation. Agronomists and computer scientists work together in order to design a kowledge based system. They focus on the representation of the spatial organisation of farm territories. This representation of a certain type of space is a collaborative activity embedded within its own space ; this collaborative activity relies on a interactional story whose resources are expertises, documents, instruments and also writing supports. Based on the paradigm of the situated and distributed cognition, our approach considers that the interaction is a dynamic process that shapes linguistic, corporal, and artefactual

\footnotetext{
* Équipe Codisant - LabPsyLor, Université Nancy 2, BP 33-97, 54015 Nancy Cedex et ICN-École de Management, 13 rue Michel Ney, Nancy. Courriel : Christian.Brassacluniv-nancy2. fr

* CEVH (ENGEES - ULP), 1 quai Koch, 67000 Strasbourg et LORIA, BP 239,

54506 Vandœuvre-lès-Nancy Cedex. Courriel : fleberlengees.u-strasbg.fr
} 
forms. We show how the mobilisation of these resources which are cognitive, corporal, documentary and material, allows the group to achieve its work. Our analysis underlines that this work articulates four spaces : the agricultural space, the group working space, the writing space, and the numerical space which is the project's horizon.

Key Words: knowledge acquisition, spatial representation, interaction, artefacts, situated and distributed cognition

* Équipe Codisant - LabPsyLor, Université Nancy 2, BP 33-97, 54015 Nancy Cedex et ICN-École de Management, 13 rue Michel Ney, Nancy. Courriel : Christian.Brassaceuniv-nancy2. fr

* CEVH (ENGEES - ULP), 1 quai Koch, 67000 Strasbourg et LORIA, BP 239,

54506 Vandœuvre-lès-Nancy Cedex. Courriel : fleber@engees.u-strasbg.fr 


\section{INTRODUCTION}

La représentation de l'espace est une activité qui peut être menée dans un cadre individuel ou collectif. Un artiste peintre, un urbaniste, un paysagiste peuvent, seuls, s'engager dans une activité de production d'une telle représentation. Cette dernière peut prendre la forme d'un matériau discursif, qu'il soit stabilisé sous forme d'un texte écrit ou qu'il soit donné à entendre sous forme orale, ou aussi d'un matériau gestuel, tel celui délivré par le mime qui figure un espace ; elle peut aussi, et c'est le cas le plus courant, être réalisée sous une forme scripturale, déposée sur un support. Bien entendu, l'hybridité de la représentation est souvent de mise. Le 'représentateur' peut concevoir sa scription comme une composition de graphismes figuratifs et textuels (pensons à la carte légendée); il peut l'accompagner de paroles, de mimiques ou autres gestes. En tout cas, il s'agit pour lui de re-présenter, de présenter à nouveau, un espace tel un paysage, un territoire ou une forme architecturale. Il n'est cependant pas rare que cette activité se déploie dans une situation où plusieurs acteurs interagissent pour créer cette représentation. Le géographe travaillera avec le graphiste, l'urbaniste avec le géomètre, le vidéaste avec l'informaticien pour élaborer une carte, un plan, une maquette, une vision en trois dimensions. On aura alors une création collective de formes, engendrée par de multiples mécanismes interactionnels. C'est ce type de processus que nous aborderons ici en nous intéressant à une situation où agronomes et informaticiens collaborent à la conception d'un certain type de représentation de l'espace agricole.

La présence d'informaticiens est justifiée par le fait que l'équipe-projet que nous étudions a pour objectif l'élaboration d'un outil d'aide à la gestion de l'espace rural. Cet outil est un dispositif numérique, destiné aux acteurs de terrain (principalement aux agronomes et secondairement aux exploitants). Sa mise au point nécessite la création de graphes 'rendant compte' de l'usage effectif du territoire par ces acteurs. La réalisation de ces graphes s'appuie sur des cartes, sur des parcellaires et sur des chorèmes (nous reviendrons plus loin sur le sens de ces termes). On a donc là une longue chaîne de formes qui relie d'une part le territoire rural en tant qu'il est l'objet de pratiques d'exploitation, et, d'autre part, le logiciel, machinerie informationnelle destinée à être utilisée par les acteurs. Entre cette 'réalité' socio-physique qu'est l'exploitation agricole et cet 'artefact' socio-technique qu'est le dispositif informatique, prend place une activité collective de conception dont une des caractéristiques est qu'elle s'ancre dans un ensemble d'espaces articulés.

L'existence d'au moins quatre éléments de cet ensemble vient rapidement à l'esprit. IL s'agit de quatre 'espaces', lieux d'effectuation des activités des différents acteurs intervenant dans le projet :

- l'espace rural façonné par les acteurs (ER),

- l'espace de travail de l'équipe-projet (ET),

- l'espace des inscriptions des formes graphiques mobilisées (EI),

- l'espace numérique de la machine (EN).

Le territoire modelé par les acteurs du terrain (ER), d'une part, et le cœur du traitement numérique (EN), d'autre part, constituent respectivement la source et l'horizon de l'ensemble du projet. La situation qui nous intéresse ici se situe entre cet amont et cet aval ; elle est une arène où l'équipe-projet développe une dynamique cognitive collective dont l'objectif est la conception de graphes constituant l'étape préparatoire à l'élaboration du logiciel d'aide. Nous envisagerons cette dynamique comme un modelage de formes sémiotiques. Cette génération 
sémiotique se réalise dans un ici et maintenant clairement défini, l'espace de travail de l'équipe-projet (ET). Abstrait du territoire, cet espace contient cependant des objets y référant directement. Il y est en effet lié grâce à des artefacts graphiques (cartes, parcellaires, chorèmes, etc.) et des textes écrits (compte rendus de visite de terrain, documents réglementaires, etc.). La mobilisation de ces artefacts constitue un des éléments-clé du travail de l'équipe. Ce dernier aboutit à des formes graphiques concrètes (croquis, dessins, schémas, etc.) accompagnées d'éléments discursifs écrits (mots, phrases, sigles, etc.) et oraux (l'ensemble des prises de paroles lors de la séance). La production de ces graphismes nécessite une inscription sur des supports, ici papier, qui constituent l'espace d'inscriptions de l'activité collective (EI).

L'étude que nous proposons ici fait apparaître une projection (au sens de précipitation) de l'espace originel (le territoire) sur un espace d'inscriptions (les feuilles mobilisées) qui est une partie de l'espace de travail où l'équipe développe son activité de conception de formes (la salle instrumentée et habitée par ses membres). Il s'agit en effet pour nous de défendre l'idée que cette activité cognitive collective s'ancre dans une matérialité qui en constitue une ressource, au sens où l'ensemble des artefacts mobilisés configurent cette dynamique de conception de formes.

L'article s'organise en deux parties. La première présente le cadre de notre étude. Nous décrivons le projet mis en place par les informaticiens et les agronomes. Nous nous attachons en particulier au processus d'acquisition de connaissances qui met en œuvre la conception de représentations graphiques variées. Nous présentons ensuite la mise en situation du groupe de travail et le mode de construction de données adapté au cadre épistémologique dans lequel nous nous plaçons, la cognition située et distribuée. Dans la seconde partie, nous effectuons l'analyse d'une séquence de l'activité d'acquisition dont nous montrons qu'elle est une construction dynamique inscrite dans un espace fait d'objets, de corps et de productions langagières.

\section{DE LA GESTION D’UNE EXPLOITATION AU SYSTÈME À BASES DE CONNAISSANCES}

\subsection{L'acquisition de connaissances,...}

Comme nous l'avons esquissé plus haut, l'équipe-projet réunit agronomes et informaticiens avec pour but le développement d'un système à bases de connaissances qui permette de représenter et d'exploiter de façon automatique des données et connaissances sur des exploitations agricoles étudiées par les agronomes. Ces données et connaissances sont de différents types : données d'enquêtes, valeurs chiffrées, cartes, synthèses écrites et graphiques. Le système construit, dénommé ROSA (pour Raisonnement sur des Organisations Spatiales Agricoles), est un système de raisonnement à partir de cas, dit RàpC (Leake, 1996 ; Lieber, 1997) : il est composé d'une base de cas, constituée des données et connaissances sur les exploitations enquêtées (chaque exploitation enquêtée constitue un ou plusieurs cas), d'une base de connaissances sur le domaine et d'un module de raisonnement. Les objectifs de ce module sont de comparer et d'adapter les connaissances liées à un cas (par exemple, une exploitation ou une partie d'exploitation source, dont on connait à la fois la structure spatiale et le fonctionnement) à un autre cas (une exploitation cible dont on ne connaît que la structure). La comparaison est établie sur des mesures de similarité entre les structures 
spatiales. L'adaptation permet de proposer une logique de fonctionnement à l'exploitation cible à partir de la logique de fonctionnement de l'exploitation source en s'appuyant sur la similarité entre les structures spatiales (Le Ber et al., 2003). L'hypothèse sous-jacente peut s'exprimer ainsi : le fonctionnement de source est au fonctionnement de cible ce que la structure de source est à la structure de cible (Lieber et Napoli, 1999). La vocation de ce système est d'aider les agronomes et, plus lointainement, les conseillers agricoles à tirer parti de modes d'exploitation mis en œuvre dans des configurations territoriales analogues.

La conception de ce système s'appuie sur une phase d'acquisition de connaissances conduite en commun par les informaticiens et les agronomes (Le Ber et al., 2002). Pour ce faire, nous avons choisi d'utiliser un formalisme proche des graphes conceptuels (Sowa, 1984), car il nous a semblé particulièrement adapté à la modélisation de structures spatiales. Cette modélisation a pour source les savoirs des agronomes à propos de l'espace rural (ER) et s'appuie sur la mobilisation, au sein de l'espace de travail (ET), de deux grands types de représentations graphiques qui constituent l'espace d'inscriptions (EI). Elle constitue l'objectif local du groupe. En effet, ces graphes sont le préalable à la transcription sous forme de code et donc à l'accès à l'espace numérique $(\mathrm{EN})$ sous forme du logiciel qui, lui, est l'objectif final du projet.

\section{2. ... une mobilisation d'inscriptions}

Les premières représentations sont des cartes, des parcellaires, des chorèmes.

- Les cartes dites d'état major (IGN) servent de support à l'analyse spatiale effectuée par les agronomes,

- Les parcellaires sont des représentations graphiques issues de relevés cadastraux qui s'appuient sur ces cartes et fournit la localisation de l'ensemble des parcelles d'une exploitation,

- Les chorèmes d'exploitation sont des représentations « géométriques » qui ont pour objectif de rendre compte du mode de gestion de l'exploitant ${ }^{1}$.

Toutes ces représentations ont été élaborées avant le début de la séance. En particulier, les chorèmes l'ont été par un des deux agronomes qui sont autour de la table. Ainsi donc, l'ensemble des graphismes que nous venons de décrire sont préexistants au travail de la séance. Nous appellerons ce type de figuration de la gestion de l'espace la «modalité 1 ».

Les secondes sont des graphes qui sont construits en temps réel. Un graphe, dit graphe d'organisation spatiale (noté GOS dans la suite), est composé de deux ensembles de sommets, l'un représente des entités spatiales, l'autre des relations. Les sommets-entités et sommetsrelations peuvent être rattachés à des concepts auxquels sont associés des propriétés. Les arêtes du graphe sont étiquetées par le rôle des entités dans la relation. Nous utilisons les graphes pour modéliser les chorèmes en supposant que :

- les objets du schéma chorématique peuvent être modélisés par des sommets-entités,

- les caractéristiques de ces objets (formes et légendes associées) peuvent être décrites par des propriétés associées aux concepts,

- l'arrangement des objets peut être représenté par des sommets-relations.

\footnotetext{
${ }^{1}$ Ces chorèmes d'exploitation sont des schémas qui s'appuient sur l'alphabet des chorèmes proposé par Brunet (1986). En toute rigueur il faudrait les appeler des schémas chorématiques d'exploitaion. Par souci de simplicité nous utilliserons ici le seul terme de 'chorème'.
} 


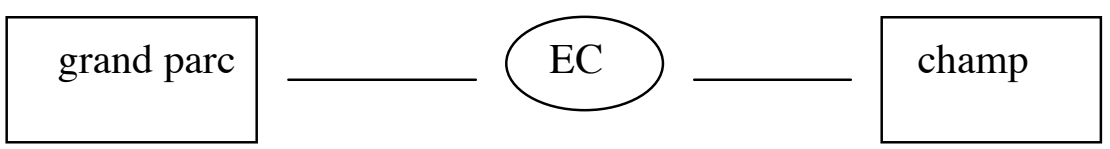

Fig. 1. Exemple de graphe d'organisation spatiale

Par exemple, le graphe ci-dessus (Fig. 1) représente deux entités (grand parc et champ) reliés par une relations de connexité ('EC' pour extérieur connexe).

Au sein de la modalité 1, les parcellaires et les chorèmes diffèrent en ce sens que les premiers conservent la topologie (positionnements relatifs des parcelles), la taille et la forme des unités de gestion de l'exploitant. Ce n'est pas tout à fait le cas des chorèmes. En effet, ceux-ci conservent grosso modo la topologie et la taille mais ils perdent la forme des parcelles et éventuellement leur nombre. On le voit très bien sur la figure suivante qui met en regard les deux types de représentation (de la «modalité $1 »$ ).

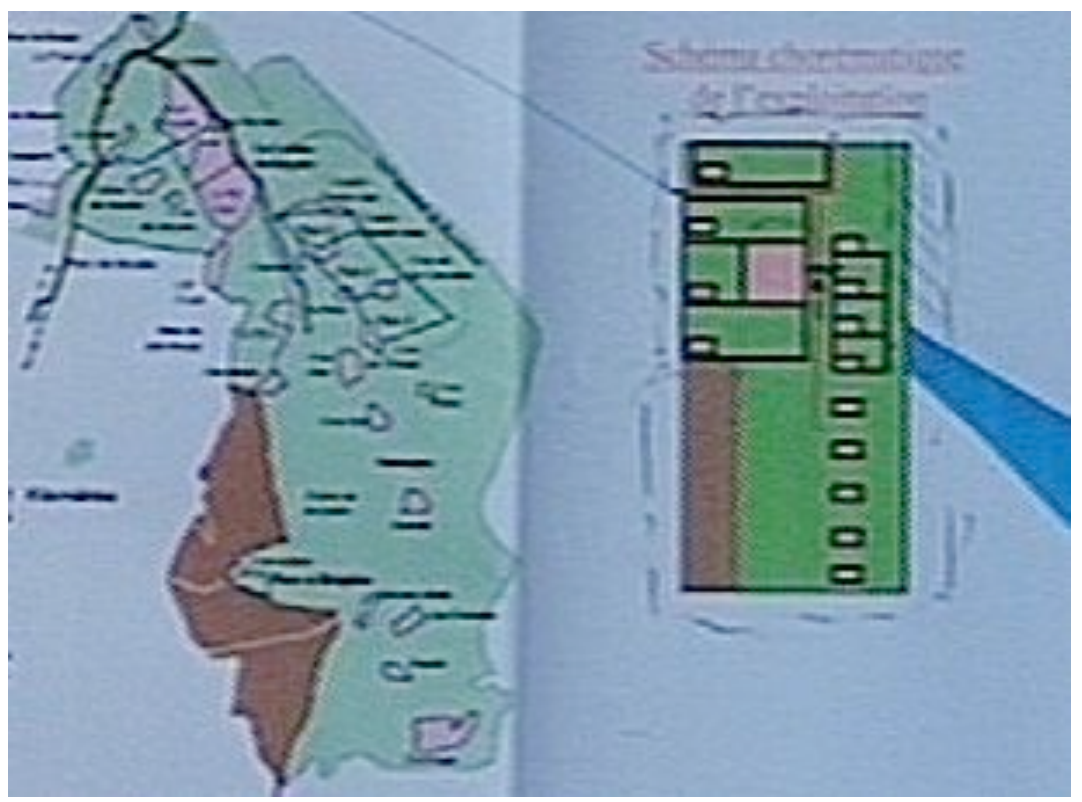

Fig. 2 : Correspondance parcellaire - chorème

La correspondance est assez évidente; elle est le travail de l'un des deux agronomes du groupe qui a, pour l'exploitation représentée ici, élaboré le chorème.

La représentation de modalité 2 , le GOS, est quant à lui le résultat d'un traçage collectif réalisé au cours de la séance de travail et conduit par Inf. La partie qui nous intéressera dans l'analyse concerne la forme qui rassemble principalement les quatre rectangles juxtaposés (dont un carré rose) correspondant à la partie haute à gauche du parcellaire et du chorème. Voici l'état de cette partie du GOS à la fin de la séquence étudiée ici (Fig. 3)2

\footnotetext{
${ }^{2}$ On note que le graphe tracé par Inf est simplifié ; il n'a pas l'orthodoxie de la figure 1. Inf ne figure pas les sommets-relations, elle ne fait que les nommer.
} 


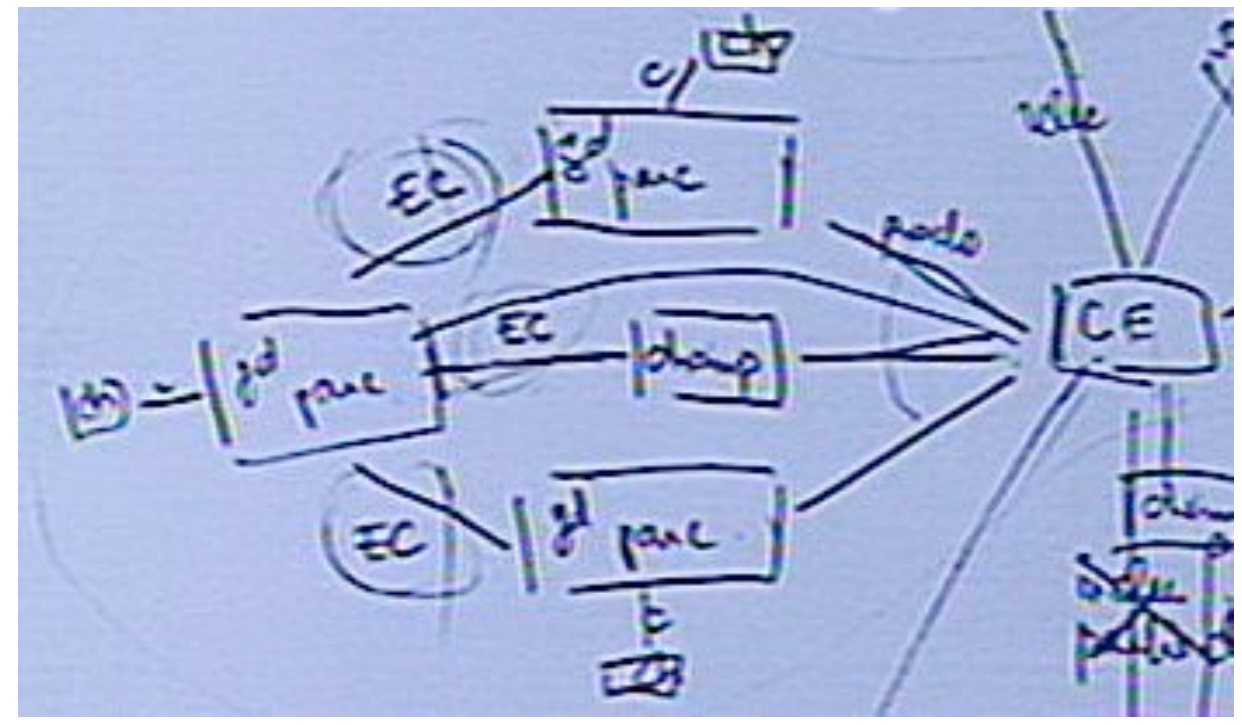

Fig. 3 : État du GOS à 38’02

\subsection{La mise en situation de l'acquisition}

Comme nous le verrons plus précisément plus loin, cette acquisition de connaissances s'appuie sur la 'traduction' des représentations graphiques d'une modalité à l'autre. Les auteurs des premières («modalité $1 »)$ sont les agronomes qui viennent en séance de travail avec les cartes, parcellaires et chorèmes. Celles relevant de la modalité 2, sont classiquement le fait du seul informaticien. Après une étape d'interrogation des experts, celui-ci produit sa modélisation censée traduire sa compréhension des dires du spécialiste, l'agronome.

Nous avons mis en place ici une situation où cette modélisation se réalise en temps réel dans le rapport effectif des deux types de 'représentateurs' et des différents graphismes. Cette volonté de mise en co-présence, à la fois des acteurs et des représentations graphiques, relève du paradigme dont nous nous réclamons. Adoptant une approche externaliste de la cognition, nous considérons l'activité d'acquisition comme étant une dynamique d'élaboration de connaissances à la fois située, ancrée dans un monde d'artefacts, et distribuée sur les entités cognitives en présence ${ }^{3}$. Les deux types d'acteurs sont co-responsables d'une dynamique de production de connaissances, configurée par les objets mobilisés (ici principalement des graphismes). Autrement dit, cette co-responsabilité s'inscrit dans une dynamique de productions langagières et gestuelles et sur une mobilisation d'objets matériels (Clark, 1997 ; Hutchins, 1995, Conein et al., 1993). Si l'une des clés de l'acquisition est son caractère dynamique, processuel, notre méthodologie de constitution de corpus doit nécessairement relever d'une clinique du processus cognitif collaboratif (Brassac, 2003). Si l'une des clés de l'acquisition est l'importance de sa multimodalité, alors un mode de construction de données s'impose : la captation vidéo.

La mise en situation est la suivante. Sous nos caméras, trois sujets travaillent conjointement, deux agronomes et une informaticienne. En début de séance ils ont sous les yeux un chorème d'une exploitation enquêtée par l'un des agronomes. Chacun d'entre eux a à sa disposition des

\footnotetext{
${ }^{3}$ Pour cet usage de la coordination des adjectifs 'situé' et 'distribué' voir (Brassac 2001, 2004).
} 
feuilles de papier (au format A4) et des instruments de traçage (crayon et stylo feutre) et de gommage. Un ensemble de documents relatifs à l'exploitation agricole en question est à portée des acteurs, immédiatement accessibles (cartes, parcellaires, etc.). La tâche qui les occupe pendant la séance est la réalisation du graphe d'occupation des sols relatif au chorème. Le filmage de la séance permet d'avoir le plan de la table de travail (filmé grâce à une caméra placée à l'aplomb) et le plan large du groupe (filmé grâce à une caméra placée face aux acteurs).

\section{TRACER CONJOINTEMENT LE GRAPHE D'OCCUPATION DES SOLS}

\subsection{Préalable}

Comme nous l'avons annoncé plus haut, nous voulons montrer en quoi l'élaboration de ce GOS est le résultat d'une activité collective ; activité collective qui s'appuie sur une interaction multimodale composée de dires et de faires. Ces dires sont des enchaînements d'énoncés proférés par les acteurs ; ces faires sont principalement des mouvements du haut du corps et des pointages et traçages qu'ils réalisent au long de la discussion (Mondada, 2004). Il s'agit donc pour nous d'analyser l'histoire qui conduit le groupe de la feuille vierge au graphique où sont consignés les entités ('CE' pour Centre d'Exploitation, 'champ' et 'gd parc') et les relations ('EC' pour extérieur connexe ${ }^{4}$, 'proche'). Cette histoire est celle du passage de la modalité 1 à la modalité 2 . La première impression que tout un chacun peut avoir en regardant les deux représentations relevant de ces deux modalités (Fig. 4) est que le GOS constitue en quelque sorte un 'éclaté' du chorème. Cet éclaté rend possible l'explicitation des relations entre les entités.

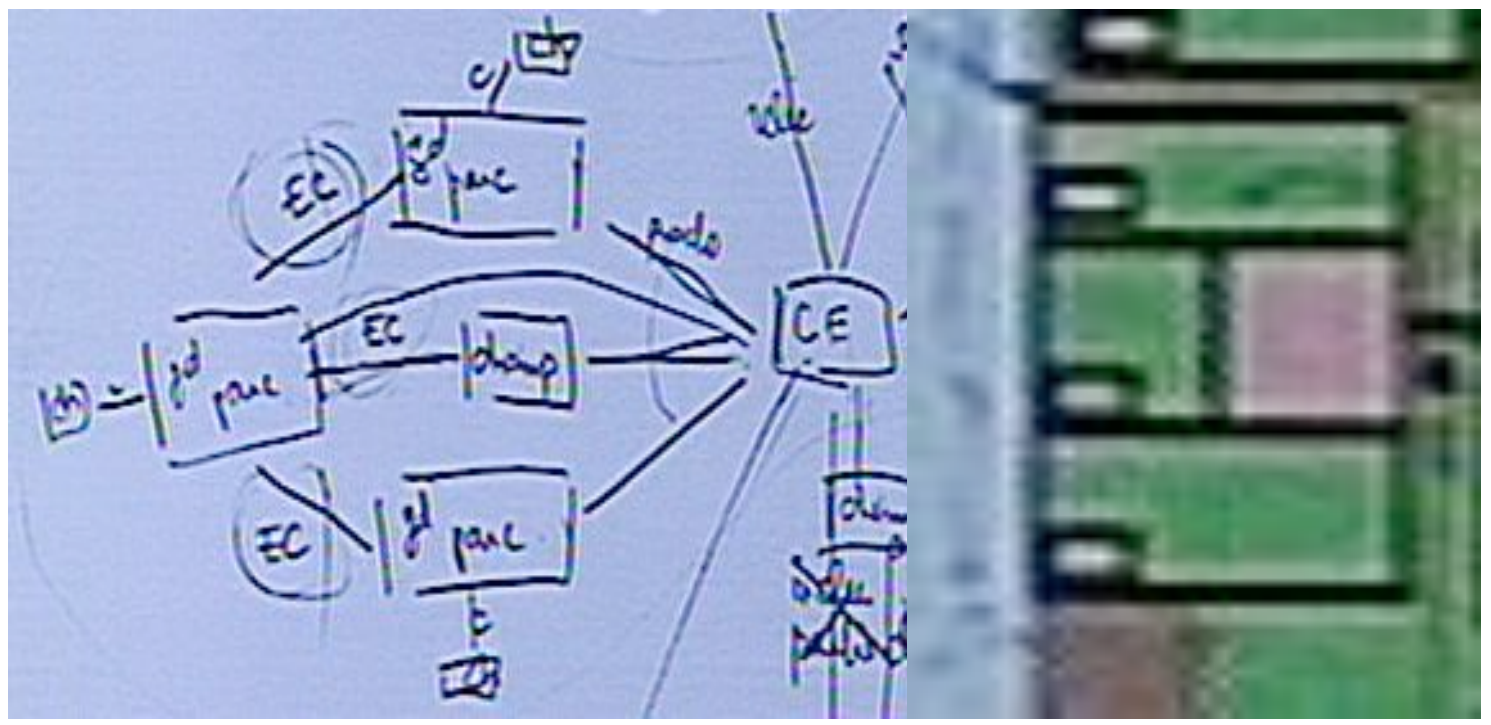

Fig. 4 : Correspondance chorème - GOS

\footnotetext{
${ }^{4}$ Deux surfaces sont dites 'extérieures connexes' si elles possèdent une frontière commune sans se recouvrir.
} 
Pour réaliser cet éclaté, l'informaticienne adopte une stratégie d'expansion par rapport au centre d'exploitation. Elle commence par tracer les trois branches radiales. Elle les nomme 'proche'; en fait elle ne le fait seulement que sur celle du haut, mais on verra plus tard que cette qualification vaut pour les deux autres (l'arc de cercle que l'on distingue le signifie graphiquement). Elle poursuit en dessinant trois rectangles (deux grands parcs et un champ, correspondant au carré rose du chorème). En disant « ce champ étant lui-même connexe à un parc $\gg\left(34^{\prime} 58 \longrightarrow 335^{\prime} 04\right)$, elle trace alors le grand parc de gauche relié à ce champ par une relation notée EC, pour «Extérieur Connexe ». On voit là que c'est Inf qui « a la main » tout en étant à l'écoute des deux agronomes. Alors qu'elle a cette intense activité de traçage sur sa feuille personnelle, elle commente de façon continue ce qu'elle fait en s'assurant du suivi de son activité par les agronomes (par exemple : «jusque là vous êtes d'accord », 35'10) et elle interagit continûment avec eux.

C'est à cet endroit que débute l'extrait que nous allons analyser et qui couvre la période 35'11-38'05. L'état de cette partie du GOS est alors le suivant :

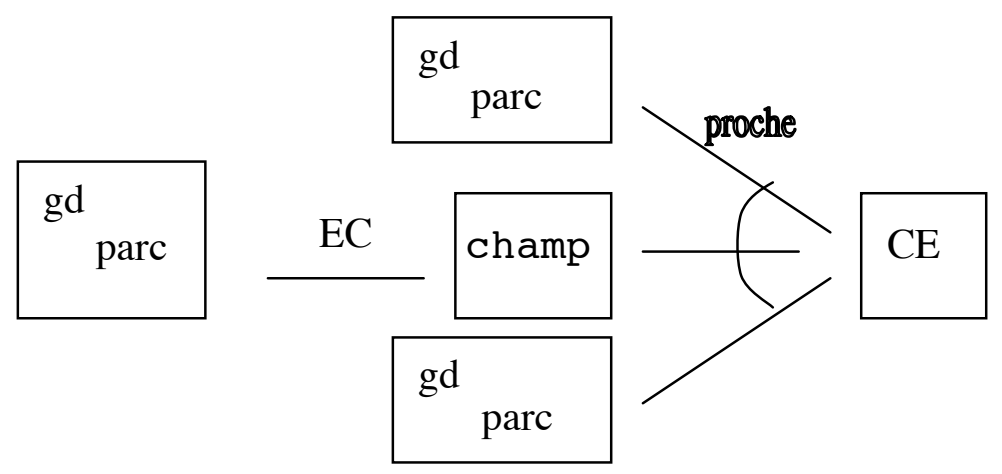

Fig. 5 : État du GOS à 35’11

Avant de débuter l'analyse à proprement parler, il nous faut donner là deux préalables, requis par une compréhension minimale de ce qui se joue dans l'interaction à trois. Ils permettent de situer dans le temps cette séquence du travail.

Premièrement, le tracé de la partie gauche du GOS fait immédiatement suite à celui de la partie droite. L'ensemble des énoncés de l'extrait, numérotés de 2 à 8 , font référence à cette partie droite. On peut voir cette partie droite sur la figure 6 qui donne l'état du GOS au moment du début de la représentation 'graphique' de la partie gauche. 


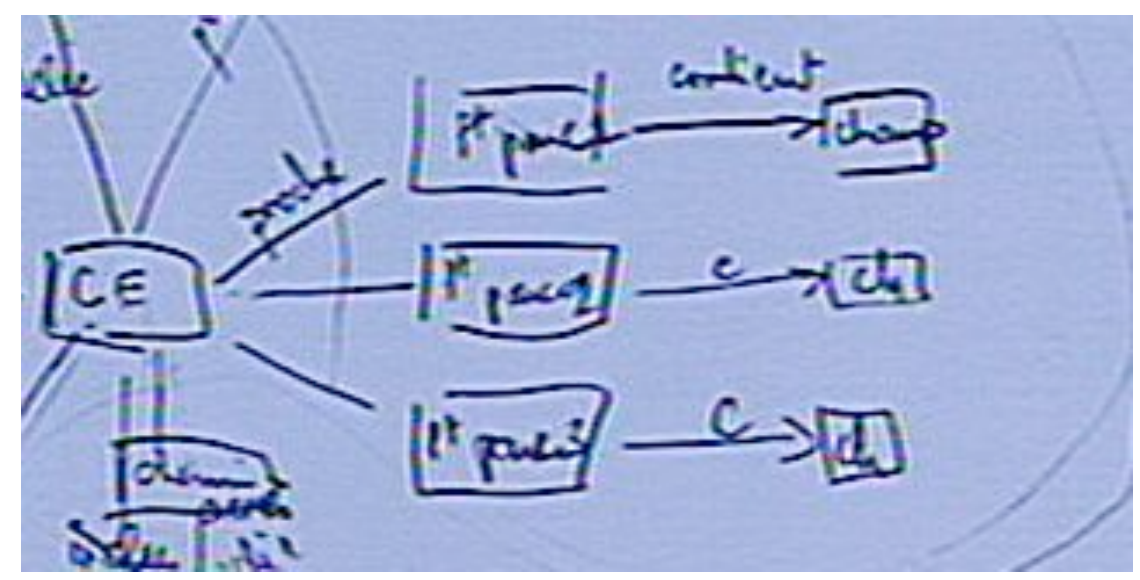

Fig. 6 : Partie droite du GOS

Deuxièmement, les acteurs impliqués dans cette activité d'acquisition travaillent lors de deux séances dans une même journée ; celle que nous étudions se déroule dans l'après-midi. Lors de la séance du matin, qui portait sur une exploitation d'une autre région française, les deux agronomes étaient présents. Ils ont échangé leur rôle entre le matin et l'après-midi. Nous appelons Cho le «chorémisateur» de la séance que nous étudions ici ; son collègue est nommé Agr. C'est la raison pour laquelle en 54, Cho dit que «ce matin on les avait pas dessinés les chemins » ('on' en tant que groupe) et qu'en 58-60, Agr affirme que "moi j'ai pas de bêtes j'ai pas j'ai pas une configuration comme ça moi effectivement moi la question que je me que je me posais c'est où passaient les animaux » ('moi' et 'je' en tant que « chorémisateur » de la séance du matin).

Trois états du GOS sont ensuite successivement produits par le groupe. Dans un premier temps, Inf relie le grand parc de gauche (qui vient d'être relié par EC au champ) aux deux autres grands parcs, également par des 'Extérieurs Connexes' (Fig. 7). Dans un second temps, Cho trace sur la feuille de Inf l'arête courbe (Fig. 8). Dans un troisième temps, Inf entoure les deux 'EC' qu'elle vient d'écrire (Fig. 9). Cet épisode est clôt par Inf à l'aide d'un geste (balayage avec le doigt au dessus de l'arête courbe) qui ne laisse pas de trace et qui valide l'état final de cette partie du GOS (« mais bon ceci dit on peut faire directement » 74-75).

\subsection{Analyse}

\subsubsection{L'extrait (35'11 - 38'05)}

Nous proposons d'abord au lecteur l'intégralité de l'extrait en nous limitant aux dires des acteurs. Nous redonnerons, au fur et à mesure de l'analyse, faite en quatre temps, la transcription de l'interaction en ajoutant cette fois-ci des éléments ayant trait aux productions gestuelles et de traçages. Voici donc simplement ce que les trois acteurs disent dans les trois minutes qui nous intéresserons.

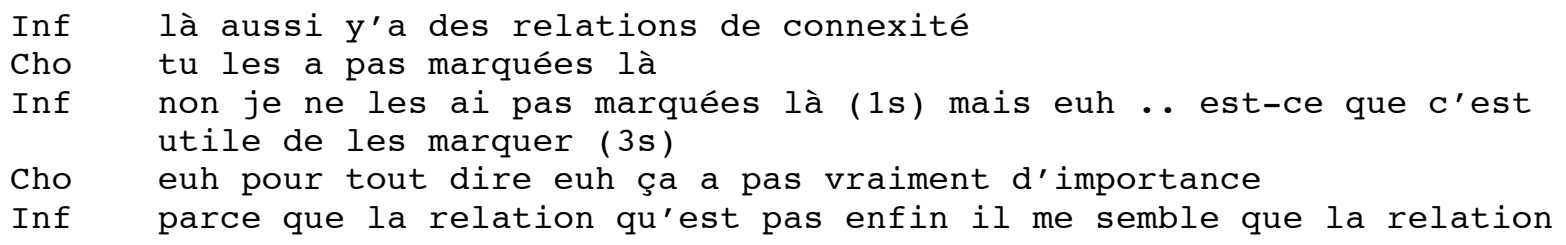




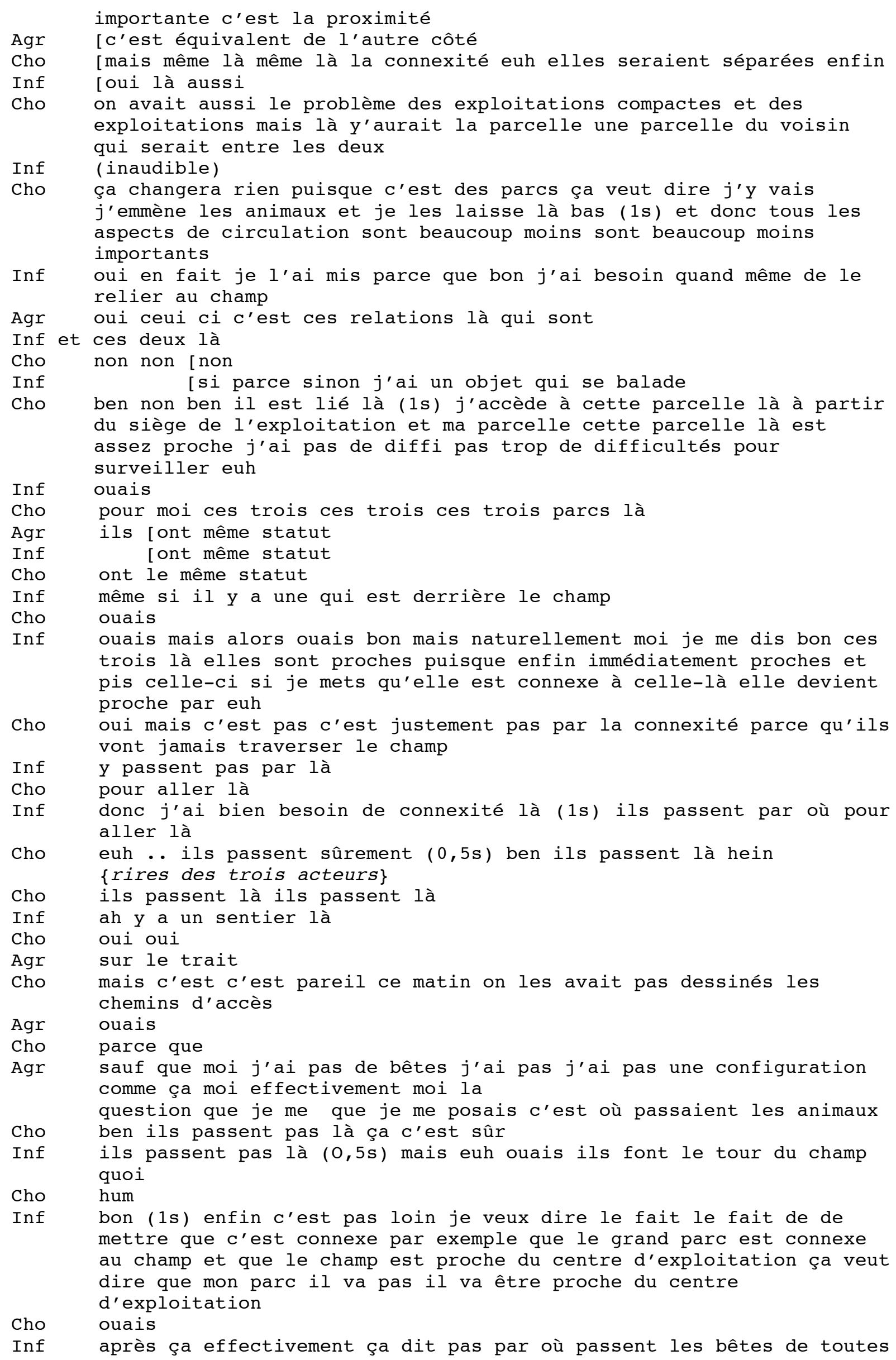




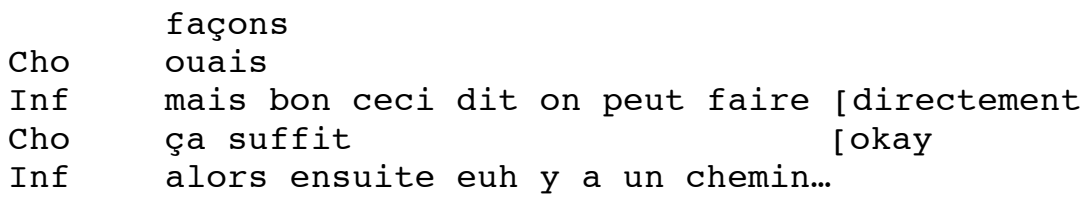

\subsubsection{Les deux extérieurs connexes (EC)}

\section{Extrait 1}

1 Inf là aussi y'a des relations de connexité \{tracé et dénomination des deux arêtes\}

Cho tu les a pas marquées là \{pointage sur partie droite du Gos\}

Inf non je ne les ai pas marquées là (1s) mais euh .. est-ce que c'est

utile de les marquer (3s) \{pointage sur partie droite du GoS\}

Cho euh pour tout dire euh ça a pas vraiment d'importance

Inf parce que la relation qu'est pas enfin il me semble que la relation

importante c'est la proximité

Agr [c'est équivalent de l'autre côté

Cho [mais même là même là la connexité euh elles seraient séparées enfin

Inf [oui là aussi

Cho on avait aussi le problème des exploitations compactes et des exploitations mais là y'aurait la parcelle une parcelle du voisin qui serait entre les deux

Inf (inaudible)

Cho ça changera rien puisque c'est des parcs ça veut dire j'y vais j'emmène les animaux et je les laisse là bas (1s) et donc tous les aspects de circulation sont beaucoup moins sont beaucoup moins importants

Inf oui en fait je l'ai mis parce que bon j'ai besoin quand même de le relier au champ

Agr oui ceui ci c'est ces relations là qui sont

Inf et ces deux là

Cho non non [non

Inf [si parce sinon j'ai un objet qui se balade

À partir de l'état du GOS montré dans la figure 5, Inf dit «là aussi y'a des relations de connexité »(1) et les trace en les nommant. Cette nouveauté apportée par Inf sur le GOS (Fig. 7) est immédiatement commentée par Cho. Inf n'a pas signifié ces connexités sur la partie droite (2, Cho : «tu les as pas marquées là »). S'ensuit une discussion entre les trois acteurs sur la nécessité de le faire sur cette partie droite $(3 \leftrightarrow 18)$. Au sortir de cette discussion, ils sont d'accord pour dire que cela n'est pas utile de noter ces 'EC' à droite. À l'énoncé interrogatif de Inf (3-4, Inf : «est-ce que c'est utile de les marquer »), Cho répond que cela n'a pas vraiment d'importance (5). En affirmant 6-7, Inf propose un argument introduit par un 'parce que' et allant dans le sens de l'affirmation de Cho. Agr va dans le même sens en soulignant l'équivalence entre les deux parties (8) et Cho développe un argumentaire pour montrer que «ça changera rien» (15). Cet accord marque le fait que le traçage de Inf semble incohérent : pourquoi traiter de deux façons différentes deux parties du chorème qui semblent équivalentes ? 


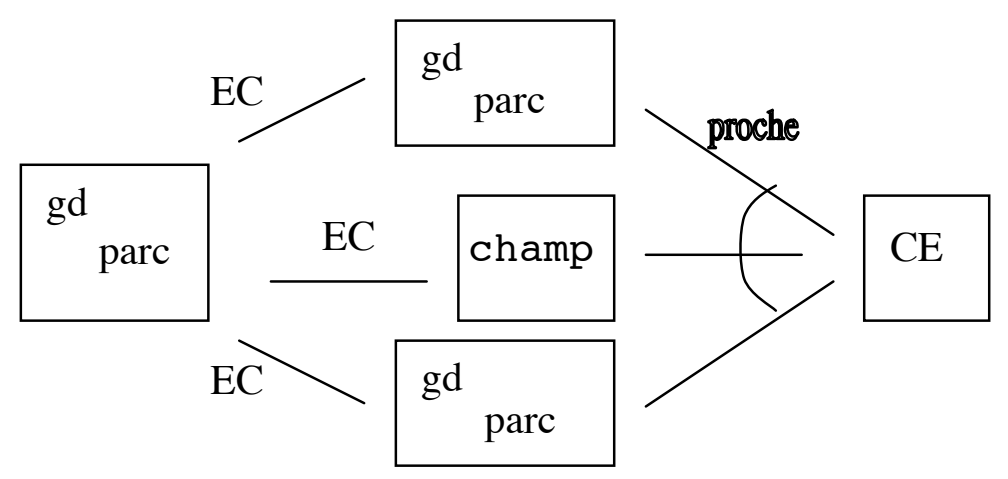

Fig. 7 : État du GOS à 35’16 (fin de la ligne 1)

C'est à cet instant (19) que Inf explique pourquoi elle a écrit ces deux 'EC'. Remarquons que cette explication a une valeur rétroactive. Tout se passe comme si, sur la base de cette discussion, elle était alors en mesure de justifier son traçage. En disant « oui en fait je l'ai mis parce que bon ... », elle donne un statut de requête à la double intervention de Cho et Agr. Ce qui apparaît comme une assertion de la part de Cho en 2, est alors en effet recyclé par Inf comme un "pourquoi tu les as marquées à gauche? » puisque entre la droite et la gauche «c'est équivalent» (8). Les interventions de Cho et Agr sont ainsi rétroactivement coordonnées via la réponse que fait Inf à la question qu'elle instancie sous cette forme : "pourquoi marques-tu les EC à gauche alors qu'il y a équivalence entre les deux côtés et que tu ne l'as pas fait à droite ?». C'est parce que ce fait, le non-marquage, est mis à jour explicitement par le pointage de Cho sur la partie droite du GOS, et implicitement par cette coordination langagière entre les deux agronomes, que Inf est amené à justifier son mode de représentation. Ce mode est ainsi mis en œuvre sous le regard, positivement critique, des agronomes. L'informaticienne explicite ce mode en 19-29 et 24. Elle le fait en utilisant un 'je', proféré deux fois au cours de 19 et répété en 24, où se glisse son identité d'informaticienne. Son horizon de travail est alors l'espace numérique (EN). La mise sous formes implantables en machine est requise par cette contingence marquée par sa 'culture' ; le GOS ne peut souffrir une entité non reliée aux autres : "j'ai besoin de le relier au champ (1920)... sinon j'ai un objet qui se balade » (24). Cette contrainte structurelle pèse en effet lourd sur son mode d'inscription. Les agronomes vont y réagir immédiatement.

\subsubsection{L'arête courbe}

\section{Extrait 2}

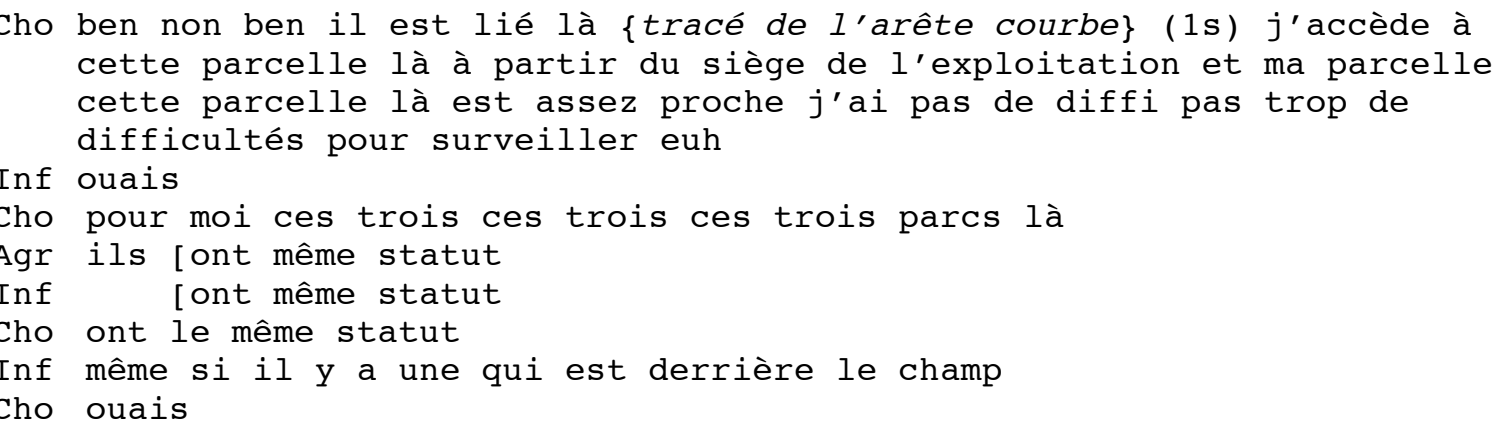


Inf ouais mais alors ouais bon mais naturellement moi je me dis bon ces trois là elles sont proches puisque enfin immédiatement proches et pis celle-ci si je mets qu'elle est connexe à celle-là elle devient proche par euh \{pointage très précis et dynamique sur le chorème\}

C'est en effet à cet instant précis que Cho fait irruption dans l'espace d'inscriptions, jusqu'à présent personnel, de Inf. Débutant son intervention par «ben non » (25), elle s'oppose à ce que vient dire l'informaticienne. En l'occurrence cette dernière vient de justifier pourquoi elle a relié le champ au grand parc de gauche par un 'EC': pour ne pas le laisser isolé. La complexité de l'action subséquente de Cho est manifeste. Au plan langagier, elle affirme qu'il (le grand parc de gauche) « est lié là ». Elle apporte ainsi une justification à son opposition. Sauf que ce 'là' est ininterprétable au seul plan discursif. En effet, ce déictique ne renvoie... qu'à ce que Cho instancie, en temps réel, via un traçage. Ce traçage, de l'arête courbe, est tout à fait concomitant avec sa prise de parole et donne une référence spatiale à ce 'là'. En quelque sorte, cette double activité langagière et gestuelle 'performatise' donc l'existence de cette liaison entre le centre d'exploitation et le grand parc de gauche. En effet, en disant «il est lié là » elle ne rend pas compte de l'état du GOS, mais en traçant simultanément l'arête courbe, elle modifie l'état de ce GOS qui devient ainsi adéquat à son dire. La multimodalité de son intervention fait advenir une relation («il est lié », 25) qui n'est pas présente dans le chorème. On voit donc ici que Inf faisait une lecture du chorème sur la base du représenté et produisait un éclaté (le GOS) traduisant les relations qui y sont lisibles. La particularité de cette arête courbe est qu'elle traduit un non dit du chorème. Là est tout l'enjeu de la suite de la discussion.

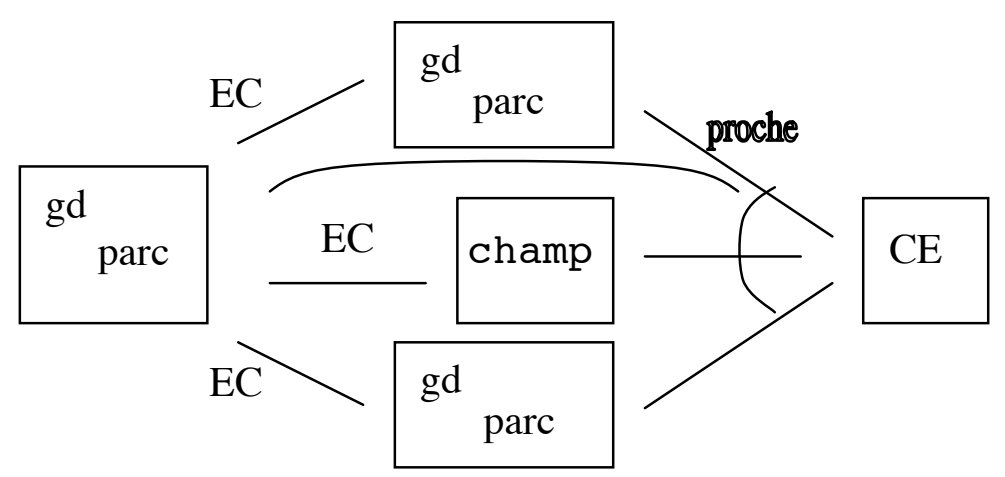

Fig. 8 : État du GOS à 36’22 (ligne25)

Cette relation est en effet doublement glosée par Cho : d'abord en termes d'accessibilité puis de proximité («j'accède à cette parcelle là (...) et cette parcelle là est assez proche », 25-2627). Ces deux caractéristiques de la relation ne sont a priori pas équivalentes (une parcelle peut être bien éloignée bien que très accessible par un bon chemin par exemple). Elles doivent être traitées par l'informaticienne qui a besoin de qualifier cette arête courbe, soudainement apparue dans l'espace d'inscriptions. Inf commence par la proximité en mobilisant la composition fonctionnelle (dont nous avons parlé plus haut) tout en apportant un petit distinguo entre 'immédiatement proche' et 'proche' : "naturellement moi je me dis bon ces trois là là elles sont proches puisque enfin immédiatement proches même et pis celle-ci si je mets qu'elle est connexe à celle-là elle devient proche par euh» $(36 \leftrightarrow 39)$. Son mode d'expression n'est pas sans évoquer la mise en mots d'un raisonnement formel. "Je me dis » marque ce 'par devers soi' de l'analyste d'un chorème devant être re-présenté de manière à 
être implantable; le «puisque» et le «si... (alors)», incomplet, illustrent le formalisme traversant l'énoncé. L'ensemble de ce discours est accompagné d'un pointage dynamique sur le chorème ayant pour fonction de rendre public les référents des déictiques. C'est alors que Cho récuse la connexité entre le champ et le grand parc de gauche : elle dit clairement «c'est justement pas par la connexité parce qu'ils vont jamais traverser le champ » (40-41-42) tout en agitant sa main et son crayon à l'adresse de Inf au moment où elle prononce ce « justement pas ». Ce faisant elle ne fait qu'expliciter une conséquence de son traçage. Le fait de tracer l'arête courbe comporte en filigrane la proposition de supprimer l' 'EC' entre le champ et le grand parc de gauche. En effet, relier directement le centre d'exploitation au grand parc de gauche rend caduque cette connexité, connexité qui assure la proximité. On a là une explicitation discursive d'un implicite artefactuel qui va conduire Inf, et plus largement le groupe, à revenir sur la question de l'accessibilité soulevée initialement par le seul Cho.

\subsubsection{L'entourage des deux 'EC'}

\section{Extrait 3}

40 Cho oui mais c'est pas c'est justement pas \{mouvement oscillant de la

41 main et du crayon dirigé vers Inf\} par la connexité parce qu'ils

42 vont jamais traverser le champ

43 Inf y passent pas par là

44 Cho pour aller là

45 Inf donc j'ai bien besoin de connexité là \{tracé des deux ellipses autour des $E C\}$

46 Cho \{pointage sur le chorème\}(1s)

47 Inf ils passent par où pour aller là \{pointage sur le chorème\}

48 Cho euh ... ils passent sûrement $(0,5 \mathrm{~s})$ ben ils passent là hein fpointage sur le parcellaire\}

$49 \quad$ \{rires des trois acteurs

50 Cho ils passent là ils passent là \{pointage très précis avec avancement du corps sur le chorème\}

51 Inf ah y a un sentier là

52 Cho oui oui

53 Agr sur le trait

En affirmant que «c'est justement pas par la connexité parce qu'ils ne vont jamais traverser le champ » (40-41-42), Cho insiste (« justement pas ») sur l'inutilité de cette connexité. Elle le fait en remettant à l'ordre du jour l'accessibilité : « ils (les animaux) vont jamais traverser le champ» (41-42). Le «y passent pas par là » (43) de Inf est produit comme un accompagnement approbatif du dire de Cho qui se termine en 44 par «pour aller là ». Un accord provisoire est donc conclu entre les deux acteurs sur cette non accessibilité par le champ... ce qui donne l'occasion à Inf d'insister sur la nécessité de figurer les deux 'EC'. En effet, si la connexité champ-grand parc de gauche n'est pas valide, il en faut bien d'autres ! Cette insistance est marquée graphiquement par ces deux entourages. Ce faisant Inf montre sa résistance à accepter cette arête courbe qui d'ailleurs n'est pas qualifiée. S'agit-il d'une arête figurant la proximité ou l'accessibilité ? La question est à cet instant encore ouverte. Rappelons encore une fois que c'est une question d'importance pour Inf qui a des contraintes d'écriture de GOS. Cho a alors un geste de pointage sur le chorème non accompagné de réplique à cette proposition de laisser ces deux 'EC'. 


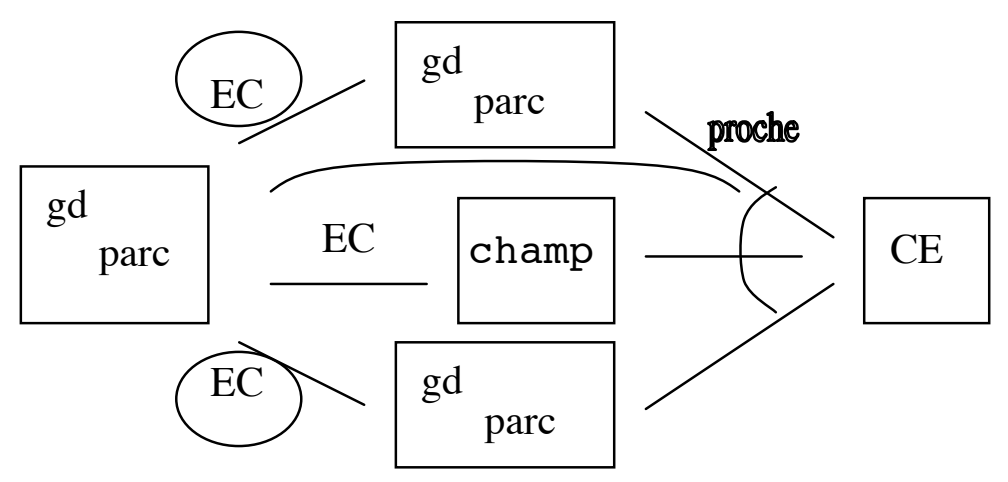

Fig. 9 : État du GOS à 37’01 (ligne 45)

Inf fait suivre ce dernier traçage par une question : «ils passent par où pour aller là » (47). L'embarras de Cho, qui a élaboré le chorème, est alors perceptible à travers plusieurs indices. Il a commencé par son silence accompagnant le pointage du chorème (46), il se poursuit par un « euh » débutant sa prise de parole, par un «sûrement » qui a la valeur habituelle de mise en doute auto centrée, par le silence suivi par un «ben » et par le rire simultané et sonore des trois acteurs. Cet embarras est aussi marqué par la mobilisation de deux types d'inscriptions en modalité 1. Il est en effet intéressant de noter que dans toute cette discussion, c'est le chorème qui sert de base au travail de conception du GOS. Tous les pointages sont faits sur lui, tous les regards convergent sur lui et le GOS. Pour la première fois Cho fait appel au parcellaire. En effet la réponse n'est pas dans le chorème ! Le référent du déictique « là » de « ils passent par là hein », 48) est situé sur le parcellaire. Les rires (des trois acteurs) accueillent cette découverte, car c'en est une. Revenant sur le chorème, Cho répète "ils passent par là » en se penchant et en pointant très précisément sur le trait qui sépare et laisse connexes le grand parc du haut et le champ rose. D'où l'énoncé de Inf (51) commençant par un «ah » d'étonnement et se terminant par «y a un sentier là », l'assentiment de Cho (52) et la précision de Agr, bien sûr ironique, « sur le trait » (53). Cho se défend en expliquant que le groupe n'avait pas dessiné les chemins d'accès dans la séance du matin. En fait à cet instant, personne n'est dupe. Le chorème ne contient pas l'information relative à la relation entre le centre d'exploitation et le grand parc de gauche. Le chorémisateur l'a fait advenir en traçant sur la feuille de Inf cette arête courbe.

\subsubsection{La validation}

\section{Extrait 4}

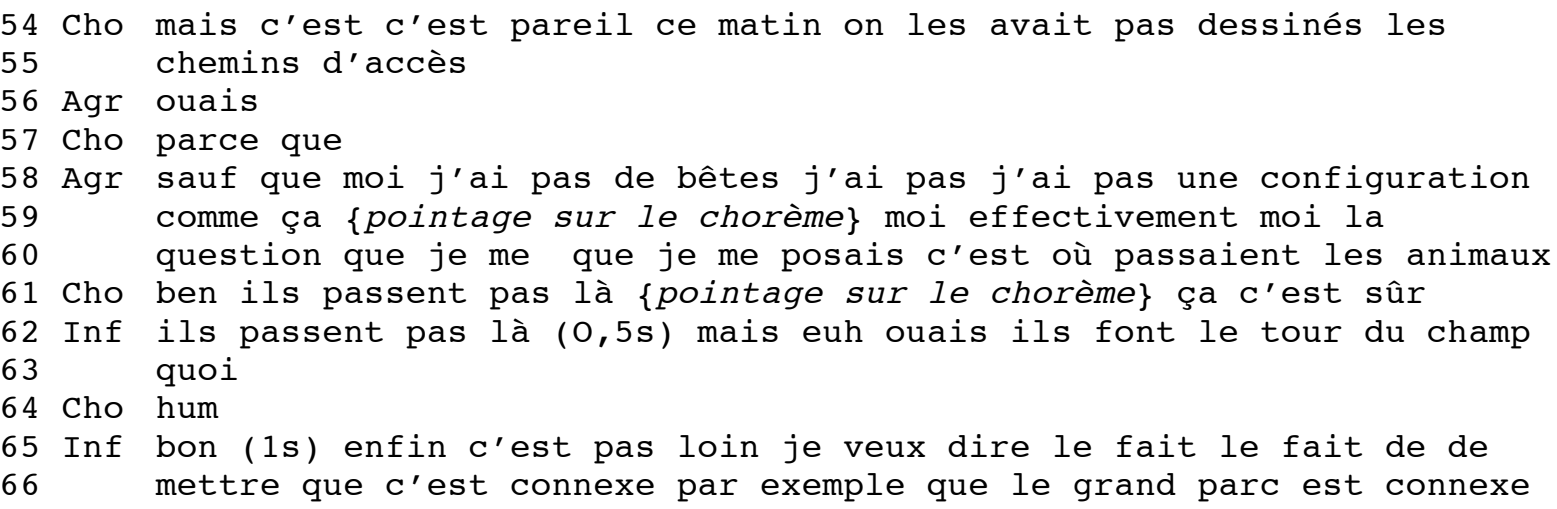




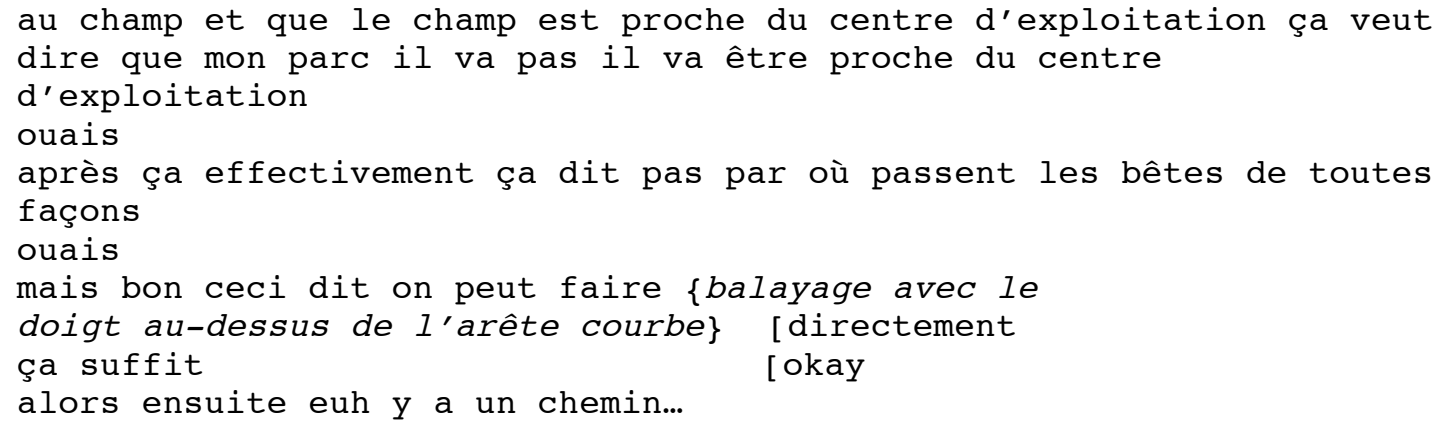

La validation de l'existence et de la nature de cette relation ne passera pas par un traçage. Alors que la discussion s'est engagée sur la question du passage des animaux dans l'exploitation travaillée lors de la séance du matin $(54 \leftrightarrow 60)$, Cho et Inf s'entendent définitivement sur le fait que les animaux contournent le champ : «ils passent pas là (par le champ) ... il font le tour du champ»(62). De fait, cette entente porte sur la question de l'accessibilité. Reste la proximité qui, rappelons-le, y était associée par Cho en 25-26-27.

À partir de l'état où se présente le GOS juste avant le tracé par Cho de l'arête courbe (Fig. 7), on peut dire que le grand parc est proche du centre d'exploitation. On peut le dire si on considère que la relation entre ces deux entités est la composée d'une proximité ('proche') et d'une connexité extérieure ('EC'). En effet si A est proche de B, lui-même connexe de C, alors A est proche de $\mathrm{C}$. La longue prise de parole de Inf $(65 \leftrightarrow 69)$ contient l'expression de cette règle. En commençant par «bon (1s) enfin c'est pas loin », elle valide d'une part le fait que l'accès est signifié par cette arête courbe (un 'bon' concessif) et elle commence d'autre part l'exposé de son sentiment eu égard à la proximité. Sentiment qu'elle explicite (« je veux dire le fait le fait de ») en déployant la composition fonctionnelle évoquée à l'instant. Son identité de modélisatrice est là encore très bien marquée. Le passage du 'je' au 'ça' par exemple est très parlant. Le 'je' du «je veux dire» marque son positionnement au sein du groupe, par rapport aux agronomes. Son rôle la conduit à modéliser une relation non présente dans le chorème et qui doit apparaître dans le GOS. Le 'ça' du «ça veut dire » est dépersonnalisé ; il s'agit d'énoncer une règle formelle. Cela dit cet énoncé n'est cependant pas d'une grande 'pureté' : on y remarque un possessif («mon parc»), on y remarque une reformulation synonymique «il va pas (être loin) il va être proche ». En tout état de cause l'explicitation de ce raisonnement reçoit un premier assentiment de Cho (70). L'accord sur la proximité étant acquis, Inf s'appuie dessus ("après ça effectivement», 71) et revient sur l'accessibilité en la secondarisant («de toutes façons», 71-72). Elle reçoit un second assentiment de la part du chorémisateur.

L'ensemble de ce double accord est finalement ratifié conjointement de manière interactionnelle et multimodale. On observe en effet une fine coordination entre Inf et Cho à la fin de cette séquence (ensuite, Inf entame la représentation d'une toute autre partie du chorème). Le « mais bon ceci dit » de Inf a une fonction de synthèse des deux questions liées à l'accessibilité et à la proximité. « On peut faire » renvoie à l'existence de l'arête courbe. Par un double aller retour, Inf balaye en effet avec le doigt juste au dessus de cette arête courbe. Alors qu'elle fait ce geste non ambigu de désignation de cette partie du GOS, Cho acquiesce une troisième fois. Elle le fait en prononçant un «ça suffit » renvoyant à la condensation en un seul représenté de la double relation d'accessibilité et de proximité. "Okay» et « directement » sont absolument concomitants. Au plan du contenu profond de l'énoncé, Inf exprime l'acceptation du traçage que Cho avait réalisé sur sa feuille personnelle. Rappelons nous en effet qu'une des fonctions de cette arête courbe était de remettre en cause l'existence 
du 'EC' écrit entre le grand parc et le champ. En disant «on peut faire directement », Inf ratifie l'existence de cette arête. Elle retient de plus le fait que ce qui est important c'est la relation directe de proximité entre le centre d'exploitation et le grand parc de gauche. Au final, l'activité de ratification de cette arête par Inf aura reçu un quadruple accord de la part de Cho ; le tout s'est passé sous le regard attentif d'Agr.

Cette partie du graphe vient de recevoir une forme stabilisée ; cette forme est le fait d'un groupe de discussion, d'un groupe d'acteurs à expertises différenciées qui ont été conduits, via une dynamique cognitive collaborative, à produire une irréversibilité pérenne, à concevoir conjointement une altération signifiante de l'espace d'inscriptions.

\section{DiSCUSSION ET CONCLUSION}

La situation d'acquisition construite ici s'écarte de la rencontre entre le modélisateur, qui a pour horizon l'espace numérique, et l'expert agronome, qui a pour source l'espace rural (et plus précisément le territoire de l'exploitation). L'informaticien n'est pas dans une situation où il s'appuie sur les dires de l'expert et sur le résultat de son travail de mise en représentation graphique (le chorème). Procéder de la sorte serait en effet supposer que la connaissance de l'agronome à propos de l'exploitation est possédée par lui. Elle serait, dans ce cadre, un état mental dont le chorème serait une projection sur le papier. Adopter une posture externaliste, non mentaliste, conduit à envisager cette connaissance comme un agi (Amin et Cohendet, 2004). Le fait de mettre en co-présence le modélisateur et deux agronomes permet d'exploiter au mieux cette inscription praxéologique du savoir du chorémisateur.

Rappelons que les agronomes ont tous les deux une pratique de production de chorèmes. La confrontation de ces deux experts autour d'un chorème permet de mettre à jour son processus d'élaboration. On aura ainsi remarqué que, dans cette séquence, ils discutent précisément de la question de l'accessibilité du grand parc à partir du centre d'exploitation. Cette discussion conduit par exemple à la nécessité (ou non) de tracer au sein du chorème un sentier le long du champ («Inf : ah y a un sentier là - Cho : « oui oui »-Agr : «sur le trait », 51 $\leftrightarrow 53$ ). Cette discussion sur le tracé, qui mobilise le parcellaire, se réalise sous les yeux de l'informaticienne ; elle est même la conséquence d'une de ses interventions ( «ils passent par où pour aller là », 47). Ainsi donc un échange de vues entre les agronomes conduit à exhiber une lacune de ce type de représentation. Cela dit, au delà de cette discussion de spécialistes des chorèmes, se joue un point beaucoup plus important vis-à-vis de la construction du graphe : la dénomination d'une entité-relation. L'analyse de l'interaction fait apparaître une différence 'culturelle' entre les chercheurs impliqués. Les outils de modélisation des agronomes d'une part et de l'informaticienne d'autre part sont de fait fondamentalement différents. Les premiers modélisent l'activité agricole à l'aide de schémas graphiques où les formes, les couleurs et les dispositions réciproques portent des significations compréhensibles par le seul truchement de l'agronome. L'alphabet symbolique des chorèmes et la légende sont nécessaires à leur lecture mais ne suffisent pas (Lardon et al., 2000 ; Capitaine et al., 2001). Les seconds représentent l'espace de façon discrète, en explicitant des entités spatiales et leurs relations, et ainsi en 'oubliant' les continuités incluses dans le chorème (d'où terme d'éclaté) ; et ce, car ils ont vocation à être traduits en lignes de code. Là où l'agronome mobilise des connaissances, le système numérique doit manipuler des informations (Foray, 2000). 
Cette différence fonctionnelle est à la fois une source de 'conflit' cognitif et une ressource pour l'équipe-projet. Le tracé du graphe se nourrit de cette différence. La preuve en est que si la distinction proche-accessible est majeure pour la modélisation à fin d'implantation et n'est que de peu d'importance pour les agronomes, elle aura nourri la réflexion contradictoire des trois acteurs et par suite aura conduit à un retraçage du graphe. Ce retraçage actualise la proposition de Cho de ne pas user de 'EC' pour la relation champ-grand parc de gauche, en conservant la seule relation 'proche'. Il est le résultat d'une co-conception conjointement validée par les deux parties.

Plutôt que de mettre en place une situation d'acquisition entre deux 'cerveaux interlocutants', nous en avons élaboré une qui rend possible une activité groupale de production de connaissances ; activité étayée sur des 'corps conversants' inscrits dans, et construisant, un monde d'artefacts. Plutôt que de supposer une responsabilité du seul expert vis-à-vis des connaissances destinées au système numérique, nous avons montré que ces connaissances adviennent au sein de la confrontation des points de vue qu'ont les uns et les autres de l'exploitation du territoire (ER) en amont, et de la machinerie computationnelle (EN) en aval. Entre l'agronome chorémisateur, l'agronome lecteur du chorème et l'informaticienne modélisatrice, se déroule une histoire cognitive qui donne lieu à une redécouverte de la teneur du schéma chorématique (le fait que cette relation entre le grand parc de gauche et le centre d'exploitation soit directe) ainsi qu'à l'élaboration du graphe. C'est l'usage, au sein de l'interaction, de cet objet-graphe (un cas typique d'objet intermédiaire au sens de Vinck, 1999) qui actualise la dissonance entre les démarches de modélisation. Le rôle de l'espace d'inscriptions (EI) est ici central. L'important est que cette dynamique de production s'inscrit à la fois dans l'intersubjectivité et dans le rapport au monde artefactuel. C'est dire que les acteurs sont co-responsables de l'histoire cognitive et que les objets font partie intégrante du processus. C'est dire que cette cognition est distribuée (sur les acteurs) et située (dans l'artefactualité). On a là une illustration de ce que humains et non humains (Latour, 1999/2004) peuvent produire en tant qu'actants d'un processus de conception de formes.

\section{RÉFÉRENCES BIBLIOGRAPHIQUES}

Brassac, C. (2004). Action située et distribuée et analyse du discours: quelques interrogations. Cahiers de Linguistique Française 26, 251-268.

Brassac, C. (2003). Éléments pour une psychologie clinique de l'activité cognitive, In M.-C. Mietkiewicz, S. Bouyer (éds), Où en est la psychologie clinique? Paris : L'Harmattan, 237-253.

Brassac, C. (2001). Formation et dialogisme: l'exemple d'un apprentissage situé et distribué, L'Orientation scolaire et professionnelle $30, \mathrm{n}^{\circ} 2,243-270$.

Brunet, R. (1986). La carte-modèle et les chorèmes. Mappemonde.

Capitaine, M., Lardon, S., Le Ber, F. \& Metzger, J.-L. (2001). Chorèmes et graphes pour modéliser les interactions entre organisation spatiale et fonctionnement des exploitations agricoles. Géomatique et espace rural. Journées CASSINI 2001, Montpellier, pp. 145-163. SIGMA, septembre 2001.

Clark, A. (1997). Being There: Putting Brain, Body, and World Together Again. Cambridge: Massachusetts Institute of Technology Press.

Amin, A., Cohendet, P. (2004). Architectures of knowledge. Firms, capabilities and communities. New York: Oxford University Press.

Conein, B., Dodier, N. \& Thévenot, L. (1993), Les objets dans l'action. De la maison au laboratoire, Paris, Éditions de l'École des Hautes Études en Sciences Sociales («Raisons pratiques »4).

Foray, D. (2000). L'économie de la connaissance. Paris : Éditions la Découverte. 
Hutchins, E. (1995). Cognition in the wild. Cambridge: Massachusetts Institute of Technology Press.

Lardon, S., Capitaine, C. \& Benoît, M. (2000). Les modèles graphiques pour représenter l'organisation spatiale des activités agricoles. In : Représentations graphiques dans les systèmes complexes naturels et artificiels. Journées de Rochebrune, pp. 127-150. ENST, janvier-février 2000.

Latour, B. (1999). Politiques de la nature. Comment faire entrer les sciences en démocratie. Paris : Éditions de la Découverte. (Réédition 2004, La Découverte/Poche).

Leake, D. B. (1996). Case-Based Reasoning. Experiences, Lessons \& Future Directions. AAAI Press/The MIT Press.

Le Ber, F., Napoli, A., Metzger, J.-L. \& Lardon, S. (2003). Modeling and comparing farm maps using graphs and case-based reasoning. Journal of Universal Computer Science, volume 9, numéro 9, 1073-1095.

Le Ber, F., Brassac, C. \& Metzger, J.-L. (2002). Analyse de l'interaction experts - informaticiens pour la modélisation de connaissances spatiales. In IC'2002, Journées Francophones d'Ingénierie des Connaissances, Rouen, pages 29-38. INSA Rouen, mai 2002.

Lieber, J. (1997). Raisonnement à partir de cas et classification hiérarchique. Application à la planification de synthèse en chimie organique. Thèse de l'Université Henri Poincaré Nancy 1, octobre 1997.

Lieber, J., Napoli, A. (1999). Raisonnement à partir de cas et résolution de problèmes dans une représentation par objets. Revue d'Intelligence Artificielle, volume 13, pp. 9-35.

Mondada, L. (2004). Temporalité, séquentialité et multimodalité au fondement de l'organisation de l'interaction : le pointage comme pratique de prise de tour. Cahiers de Linguistique Française 26, 269-292.

Sowa, J.F. (1984). Conceptual Structures: Information Processing in Mind and Machine. Paris : Addison Wesley.

Vinck, D. (1999). Les objets intermédiaires dans les réseaux de coopération scientifique. Contribution à la prise en compte des objets dans les dynamiques sociales. Revue Française de Sociologie XL (2), 385-414. 